\title{
OPTICAL SOLITON PERTURBATION AND POLARIZATION WITH QUADRATIC-CUBIC NONLINEARITY BY SINE-GORDON EQUATION APPROACH
}

\author{
Y. Yıldırım ${ }^{1}$ (D), E. Topkara ${ }^{2}$, A. Biswas ${ }^{3,4,5,6 \text { D }, \text { H. Triki }}{ }^{7}$, M. Ekici ${ }^{8}$, P. Guggilla ${ }^{3}$, S. Khan $^{3}$ iD, M. R. Belic ${ }^{9}$ \\ ${ }^{1}$ Department of Mathematics, Faculty of Arts and Sciences, Near East University, 99138 Nicosia, Cyprus \\ ${ }^{2}$ Department of Mathematics, Huston-Tillotson University, Austin, TX-78702, USA \\ ${ }^{3}$ Department of Physics, Chemistry and Mathematics, Alabama A63 University, Normal, AL 35762-4900, USA \\ ${ }^{4}$ Mathematical Modeling and Applied Computation (MMAC) Research Group, \\ Department of Mathematics, King Abdulaziz University, Jeddah-21589, Saudi Arabia \\ ${ }^{5}$ Department of Applied Mathematics, National Research Nuclear University, \\ 31, Kashirskoe Hwy, Moscow-115409, Russian Federation \\ ${ }^{6}$ Department of Mathematics and Applied Mathematics, Sefako Makgatho Health Sciences University, \\ Medunsa-0204, Pretoria, South Africa \\ ${ }^{7}$ Radiation Physics Laboratory, Department of Physics, Faculty of Sciences, \\ Badji Mokhtar University, P. O. Box 12, 23000 Annaba, Algeria \\ ${ }^{8}$ Department of Mathematics, Faculty of Science and Arts, Yozgat Bozok University, 66100 Yozgat, Turkey \\ ${ }^{9}$ Institute of Physics Belgrade, Pregrevica, 118, 11080 Zemun, Serbia, \\ e-mail: biswas.anjan@gmail.com
}

(Received 11 December 2020; in final form 17 February 2021; accepted 19 February 2021; published online 31 May 2021)

This paper recovers a full spectrum of optical solitons that are generated by the combined effects of dispersion and nonlinearity of the pulse propagation. The quadratic-cubic form of the nonlinear refractive index is incorporated in the governing nonlinear Schrödinger equation, which governs the dynamics of the soliton transmission across trans-continental and transoceanic distances. The model is considered with a nonlinear chromatic dispersion that is required to sustain for smooth transmission of soliton pulses in optical fibers, couplers, PCF, magneto-optic waveguides, crystals, metamaterials, metasurfaces, birefringent fibers, DWDM systems and other form of waveguides. Solitons in birefringent fibers as well as solitons in polarization preserving fibers are considered. The governing model is treated with Hamiltonian type perturbation terms. The perturbation terms are with full intensity. The model is studied for the intensity count $m=1$. The adopted integration algorithm is the sine-Gordon equation method that reveals single form soliton solutions as well as dual-form soliton solutions. These solitons are dark soliton, singular soliton, bright soliton and combo singular soliton. Also, dark soliton represents a kink/anti-kink solitary wave or a shock wave in fluid dynamics. The respective constraint conditions are also in place to guarantee the existence of such solitons.

Key words: solitons, polarization, perturbation, quadratic-cubic nonlinearity.

DOI: https://doi.org/10.30970/jps.25.2001

\section{INTRODUCTION}

Optical solitons with the quadratic-cubic (QC) form of the nonlinear refractive index have attracted attention during the past couple of decades [1-10]. Such a form of the nonlinear refractive index first appeared during 1994 and later was rejuvenated in 2011 [7, 8]. Subsequently, a deluge of results ensued. These stem from addressing the model from a variety of angles. These are semi-inverse variational principle, mapping methods, $G^{\prime} / G$-scheme, collective variables, extended trial function, extended Jacobi's elliptic function expansion, F-expansion scheme, stationary solitons with nonlinear chromatic-dispersion, soliton perturbation theory, magneto optic waveguides, Bragg gratings and many more [1-10, 17-20]. There are still a lot of unknown facts that are yet to be uncovered. This paper will apply the sine-Gordon equation approach to reveal a spectrum of soliton solutions that are of a single form as well as dual form. In this context, soliton polarization in birefringent fibers as well as solitons in polarization preserving fibers are considered. There are a few perturbati- on terms that are also incorporated in the governing nonlinear Schrödinger equation (NLSE) that appear with maximum intensity but stay below their critical count. The details are studied in the rest of the paper in two subsequent sections that focus on polarization preserving fibers, and birefringent fibers respectively.

\section{POLARIZATION PRESERVING FIBERS}

The NLSE with QC nonlinearity for polarizationpreserving fibers is:

$$
i q_{t}+a q_{x x}+\left(b_{1}|q|+b_{2}|q|^{2}\right) q=0,
$$

where $x$ and $t$ are the non-dimensional distance and time in a dimensionless form, respectively. The first term is linear temporal evolution and $i=\sqrt{-1}$. The complex valued function $q(x, t)$ represents optical solitons in polarization-preserving fibers. The constant $a$ is the coefficient of chromatic dispersion (CD), while the coefficients $b_{1}$ and $b_{2}$ constitute quadratic-cubic nonlinearity. 
In the presence of perturbation terms, NLSE with QC nonlinearity for polarization-preserving fibers reads:

$$
\begin{aligned}
& i q_{t}+a q_{x x}+\left(b_{1}|q|+b_{2}|q|^{2}\right) q \\
& =i\left[\alpha\left(|q|^{2 m} q\right)_{x}+\lambda\left(|q|^{2 m}\right)_{x} q+\mu|q|^{2 m} q_{x}\right],
\end{aligned}
$$

where the constant $\alpha$ represents the coefficient of selfsteepening nonlinearity, while the constants $\lambda$ and $\mu$ are the coefficients of the higher-order dispersion effects. Also, $m$ accounts for the maximum intensity count.

To obtain the soliton solution, we set

$$
\begin{gathered}
q(x, t)=U(\xi) e^{i \varphi(x, t)}, \quad \xi=\eta(x-v t), \\
\varphi(x, t)=-\kappa x+\omega t+\theta_{0},
\end{gathered}
$$

where $\kappa, v, \omega, \eta$ and $\theta_{0}$ are, respectively, the frequency, velocity, wave number, width and the phase constant of the soliton. Also, the functions $U(\xi)$ and $\varphi(x, t)$ are the amplitude and the phase component of the soliton, respectively.

Inserting Eq. (3) into Eq. (2) yields the real equation

$$
\begin{aligned}
a \eta^{2} U^{\prime \prime} & -\left(\omega+a \kappa^{2}\right) U+b_{1} U^{2}+b_{2} U^{3} \\
& -\kappa(\alpha+\mu) U^{2 m+1}=0
\end{aligned}
$$

and the imaginary equation

$$
v+2 a \kappa+(2 \alpha m+2 \lambda m+\alpha+\mu) U^{2 m}=0,
$$

where the velocity of the soliton is

$$
v=-2 a \kappa
$$

while the constraint relation between the perturbation terms is

$$
2 \alpha m+2 \lambda m+\alpha+\mu=0 .
$$

Equation (4) can be integrated to determine the soliton profile. According to the sine-Gordon equation method, Eq. (4) satisfies

$$
U(\xi)=\sum_{i=1}^{N} \cos ^{i-1}(V(\xi))\left[\begin{array}{c}
B_{i} \sin (V(\xi)) \\
+A_{i} \cos (V(\xi))
\end{array}\right]+A_{0},
$$

where $A_{i}$ and $B_{i}(0 \leq i \leq N)$ are constants, $N$ is the balance number and $V(\xi)$ holds

$$
V^{\prime}(\xi)=\sin (V(\xi))
$$

with

$$
\begin{aligned}
& \sin (V(\xi))=\operatorname{sech}(\xi), \\
& \sin (V(\xi))=i \operatorname{csh}(\xi), \\
& \cos (V(\xi))=\tanh (\xi), \\
& \cos (V(\xi))=\operatorname{coth}(\xi) .
\end{aligned}
$$

By using the balancing principle in Eq. (4), Eq. (8) reduces to

$$
U(\xi)=B_{1} \sin (V(\xi))+A_{1} \cos (V(\xi))+A_{0} .
$$

Substituting Eq. (11) with Eq. (9) into Eq. (4) leads to

\section{Case 1:}

$$
\begin{aligned}
& m=1, \quad \eta= \pm \sqrt{\frac{a \kappa^{2}+\omega}{4 a}}, \\
& A_{0}= \pm \sqrt{\frac{a \kappa^{2}+\omega}{2\left(\alpha \kappa+\kappa \mu-b_{2}\right)}}, \\
& A_{1}= \pm \sqrt{\frac{a \kappa^{2}+\omega}{2\left(\alpha \kappa+\kappa \mu-b_{2}\right)}}, \quad B_{1}=0, \\
& b_{1}= \pm \sqrt{\frac{9\left(a \kappa^{2}+\omega\right)\left(\alpha \kappa+\kappa \mu-b_{2}\right)}{2}} .
\end{aligned}
$$

Plugging Eq. (12) with Eq. (10) into Eq. (11) leads to the dark soliton

$$
q(x, t)=\left\{ \pm \sqrt{\frac{a \kappa^{2}+\omega}{2\left(\alpha \kappa+\kappa \mu-b_{2}\right)}} \pm \sqrt{\frac{a \kappa^{2}+\omega}{2\left(\alpha \kappa+\kappa \mu-b_{2}\right)}} \tanh \left[\sqrt{\frac{a \kappa^{2}+\omega}{4 a}}(x+2 a \kappa t)\right]\right\} e^{i\left(-\kappa x+\omega t+\theta_{0}\right)}
$$

and the singular soliton

$$
q(x, t)=\left\{ \pm \sqrt{\frac{a \kappa^{2}+\omega}{2\left(\alpha \kappa+\kappa \mu-b_{2}\right)}} \pm \sqrt{\frac{a \kappa^{2}+\omega}{2\left(\alpha \kappa+\kappa \mu-b_{2}\right)}} \operatorname{coth}\left[\sqrt{\frac{a \kappa^{2}+\omega}{4 a}}(x+2 a \kappa t)\right]\right\} e^{i\left(-\kappa x+\omega t+\theta_{0}\right)} .
$$


These solitons are valid for

$$
\begin{aligned}
\left(\alpha \kappa+\kappa \mu-b_{2}\right)\left(a \kappa^{2}+\omega\right) & >0 \\
a\left(a \kappa^{2}+\omega\right) & >0 .
\end{aligned}
$$

It must be noted that solutions (13) and (14) are dark and singular solitons, respectively, in nonlinear fiber optics. However, solution (13) is referred to as kink/anti-kink or shock wave in fluid dynamics.

\section{Case 2:}

$$
\begin{aligned}
& m=1, \quad \eta= \pm \sqrt{-\frac{a \kappa^{2}+\omega}{2 a}}, \\
& A_{0}= \pm \sqrt{\frac{a \kappa^{2}+\omega}{2\left(\alpha \kappa+\kappa \mu-b_{2}\right)}}, \quad A_{1}=0 \\
& B_{1}= \pm \sqrt{\frac{a \kappa^{2}+\omega}{\alpha \kappa+\kappa \mu-b_{2}},} \\
& b_{1}= \pm \sqrt{\frac{9\left(a \kappa^{2}+\omega\right)\left(\alpha \kappa+\kappa \mu-b_{2}\right)}{2}} .
\end{aligned}
$$

Inserting Eq. (17) with Eq. (10) into Eq. (11) causes to the bright soliton

$$
q(x, t)=\left\{ \pm \sqrt{\frac{a \kappa^{2}+\omega}{2\left(\alpha \kappa+\kappa \mu-b_{2}\right)}} \pm \sqrt{\frac{a \kappa^{2}+\omega}{\alpha \kappa+\kappa \mu-b_{2}}} \operatorname{sech}\left[\sqrt{-\frac{a \kappa^{2}+\omega}{2 a}}(x+2 a \kappa t)\right]\right\} e^{i\left(-\kappa x+\omega t+\theta_{0}\right)}
$$

The bright soliton is valid for the constraint (15) along with

$$
a\left(a \kappa^{2}+\omega\right)<0
$$

\section{Case 3:}

$$
\begin{aligned}
& m=1, \quad \eta= \pm \sqrt{\frac{a \kappa^{2}+\omega}{a}}, \\
& A_{0}= \pm \sqrt{\frac{a \kappa^{2}+\omega}{2\left(\alpha \kappa+\kappa \mu-b_{2}\right)}}, \\
& A_{1}= \pm \sqrt{\frac{a \kappa^{2}+\omega}{2\left(\alpha \kappa+\kappa \mu-b_{2}\right)}}, \\
& B_{1}= \pm \sqrt{\frac{a \kappa^{2}+\omega}{2\left(\alpha \kappa+\kappa \mu-b_{2}\right)}}, \\
& b_{1}= \pm \sqrt{\frac{9\left(a \kappa^{2}+\omega\right)\left(\alpha \kappa+\kappa \mu-b_{2}\right)}{2}} .
\end{aligned}
$$

Substituting Eq. (20) with Eq. (10) into Eq. (11) yields the combo singular soliton

$$
\begin{aligned}
q(x, t) & =\left\{ \pm \sqrt{\frac{a \kappa^{2}+\omega}{2\left(\alpha \kappa+\kappa \mu-b_{2}\right)}} \pm \sqrt{\frac{a \kappa^{2}+\omega}{2\left(\alpha \kappa+\kappa \mu-b_{2}\right)}} \operatorname{coth}\left[\sqrt{\frac{a \kappa^{2}+\omega}{a}}(x+2 a \kappa t)\right] \pm \sqrt{\frac{a \kappa^{2}+\omega}{2\left(\alpha \kappa+\kappa \mu-b_{2}\right)}}\right. \\
& \left.\times \operatorname{csch}\left[\sqrt{\frac{a \kappa^{2}+\omega}{a}}(x+2 a \kappa t)\right]\right\} e^{i\left(-\kappa x+\omega t+\theta_{0}\right)} .
\end{aligned}
$$

The combo singular soliton is valid for the constraints (15) and (16). 


\section{BIREFRINGENT FIBERS}

\section{A. Unperturbed model}

The coupled system derived from the equation (1) for birefringent fibers with four-wave mixing ( $4 \mathrm{WM})$ is:

$$
\begin{aligned}
i q_{t} & +a_{1} q_{x x}+b_{1} q \sqrt{|q|^{2}+|r|^{2}+q r^{*}+q^{*} r} \\
& +\left(c_{1}|q|^{2}+d_{1}|r|^{2}\right) q+e_{1} r^{2} q^{*}=0, \\
i r_{t} & +a_{2} r_{x x}+b_{2} r \sqrt{|r|^{2}+|q|^{2}+r q^{*}+r^{*} q} \\
& +\left(c_{2}|r|^{2}+d_{2}|q|^{2}\right) r+e_{2} q^{2} r^{*}=0,
\end{aligned}
$$

where the complex valued functions $q(x, t)$ and $r(x, t)$ account for optical solitons in birefringent fibers. For $l=1,2, a_{l}$ are the coefficients of $\mathrm{CD}$ and $c_{l}$ represent the coefficients of self-phase modulation (SPM), while $d_{l}$ account for the coefficients of cross-phase modulation (XPM) and $e_{l}$ stand for the coefficients of $4 \mathrm{WM}$. For the coefficients $b_{l}$, the first two terms are SPM and XPM, respectively, while the last two are $4 \mathrm{WM}$ inside the radical sign.

To obtain the soliton solution, we set

$$
\begin{aligned}
& q(x, t)=U_{1}(\xi) e^{i \varphi(x, t)}, \quad r(x, t)=U_{2}(\xi) e^{i \varphi(x, t)}, \\
& \xi=\eta(x-v t), \quad \varphi(x, t)=-\kappa x+\omega t+\theta_{0} .
\end{aligned}
$$

Inserting Eq. (24) into Eqs. (22) and (23) yields the real equation

$$
\begin{aligned}
& a_{l} \eta^{2} U_{l}^{\prime \prime}-\left(\omega+a_{l} \kappa^{2}\right) U_{l}+b_{l} U_{l}^{2}+b_{l} U_{l} U_{\tilde{l}} \\
& +c_{l} U_{l}^{3}+\left(d_{l}+e_{l}\right) U_{l} U_{\tilde{l}}^{2}=0
\end{aligned}
$$

and the imaginary equation

$$
v=-2 a_{l} \kappa
$$

where $j=1,2$ and $\tilde{j}=3-j$. Equation (25) reduces to

$$
\begin{aligned}
& a_{l} \eta^{2} U_{l}^{\prime \prime}-\left(\omega+a_{l} \kappa^{2}\right) U_{l} \\
& +2 b_{l} U_{l}^{2}+\left(c_{l}+d_{l}+e_{l}\right) U_{l}^{3}=0
\end{aligned}
$$

by the constraint $U_{\tilde{l}}=U_{l}$. By using the balancing principle in Eq. (27), Eq. (8) reduces to

$$
U_{l}(\xi)=B_{1} \sin \left(V_{l}(\xi)\right)+A_{1} \cos \left(V_{l}(\xi)\right)+A_{0}
$$

Substituting Eq. (28) with Eq. (9) into Eq. (27) leads to

\section{Case 1:}

$$
\begin{aligned}
& \eta= \pm \sqrt{\frac{a_{l} \kappa^{2}+\omega}{4 a_{l}},} \\
& A_{0}= \pm \sqrt{-\frac{a_{l} \kappa^{2}+\omega}{2\left(c_{l}+d_{l}+e_{l}\right)}}, \quad B_{1}=0, \\
& A_{1}= \pm \sqrt{-\frac{a_{l} \kappa^{2}+\omega}{2\left(c_{l}+d_{l}+e_{l}\right)}}, \\
& b_{l}= \pm \sqrt{-\frac{9\left(a_{l} \kappa^{2}+\omega\right)\left(c_{l}+d_{l}+e_{l}\right)}{8}} .
\end{aligned}
$$

Plugging Eq. (29) with Eq. (10) into Eq. (28) leads to the dark solitons

$$
\begin{aligned}
& q(x, t)=\left\{ \pm \sqrt{-\frac{a_{1} \kappa^{2}+\omega}{2\left(c_{1}+d_{1}+e_{1}\right)}} \pm \sqrt{-\frac{a_{1} \kappa^{2}+\omega}{2\left(c_{1}+d_{1}+e_{1}\right)}} \tanh \left[\sqrt{\frac{a_{1} \kappa^{2}+\omega}{4 a_{1}}}\left(x+2 a_{1} \kappa t\right)\right]\right\} e^{i\left(-\kappa x+\omega t+\theta_{0}\right)}, \\
& r(x, t)=\left\{ \pm \sqrt{-\frac{a_{2} \kappa^{2}+\omega}{2\left(c_{2}+d_{2}+e_{2}\right)}} \pm \sqrt{-\frac{a_{2} \kappa^{2}+\omega}{2\left(c_{2}+d_{2}+e_{2}\right)}} \tanh \left[\sqrt{\frac{a_{2} \kappa^{2}+\omega}{4 a_{2}}}\left(x+2 a_{2} \kappa t\right)\right]\right\} e^{i\left(-\kappa x+\omega t+\theta_{0}\right)}
\end{aligned}
$$

and the singular solitons

$$
\begin{aligned}
& q(x, t)=\left\{ \pm \sqrt{-\frac{a_{1} \kappa^{2}+\omega}{2\left(c_{1}+d_{1}+e_{1}\right)}} \pm \sqrt{-\frac{a_{1} \kappa^{2}+\omega}{2\left(c_{1}+d_{1}+e_{1}\right)}} \operatorname{coth}\left[\sqrt{\frac{a_{1} \kappa^{2}+\omega}{4 a_{1}}}\left(x+2 a_{1} \kappa t\right)\right]\right\} e^{i\left(-\kappa x+\omega t+\theta_{0}\right)}, \\
& r(x, t)=\left\{ \pm \sqrt{-\frac{a_{2} \kappa^{2}+\omega}{2\left(c_{2}+d_{2}+e_{2}\right)}} \pm \sqrt{-\frac{a_{2} \kappa^{2}+\omega}{2\left(c_{2}+d_{2}+e_{2}\right)}} \operatorname{coth}\left[\sqrt{\frac{a_{2} \kappa^{2}+\omega}{4 a_{2}}}\left(x+2 a_{2} \kappa t\right)\right]\right\} e^{i\left(-\kappa x+\omega t+\theta_{0}\right)}
\end{aligned}
$$

Again, in nonlinear optics solutions (30)-(31) are referred to as a dark-dark soliton pair while in fluids they represent 
a kink/anti-kink solitary wave or a shock wave pair. These solitons are valid for

$$
\begin{aligned}
\left(c_{l}+d_{l}+e_{l}\right)\left(a_{l} \kappa^{2}+\omega\right) & <0, \\
a_{l}\left(a_{l} \kappa^{2}+\omega\right) & >0 .
\end{aligned}
$$

\section{Case 2:}

$$
\begin{aligned}
& \eta= \pm \sqrt{-\frac{a_{l} \kappa^{2}+\omega}{2 a_{l}},} \\
& A_{0}= \pm \sqrt{-\frac{a_{l} \kappa^{2}+\omega}{2\left(c_{l}+d_{l}+e_{l}\right)}}, \quad A_{1}=0, \\
& B_{1}= \pm \sqrt{-\frac{a_{l} \kappa^{2}+\omega}{c_{l}+d_{l}+e_{l}},} \\
& b_{l}= \pm \sqrt{-\frac{9\left(a_{l} \kappa^{2}+\omega\right)\left(c_{l}+d_{l}+e_{l}\right)}{8}} .
\end{aligned}
$$

Inserting Eq. (36) with Eq. (10) into Eq. (28) leads to the bright solitons

$$
\begin{aligned}
& q(x, t)=\left\{ \pm \sqrt{-\frac{a_{1} \kappa^{2}+\omega}{2\left(c_{1}+d_{1}+e_{1}\right)}} \pm \sqrt{-\frac{a_{1} \kappa^{2}+\omega}{c_{1}+d_{1}+e_{1}}} \operatorname{sech}\left[\sqrt{-\frac{a_{1} \kappa^{2}+\omega}{2 a_{1}}}\left(x+2 a_{1} \kappa t\right)\right]\right\} e^{i\left(-\kappa x+\omega t+\theta_{0}\right)}, \\
& r(x, t)=\left\{ \pm \sqrt{-\frac{a_{2} \kappa^{2}+\omega}{2\left(c_{2}+d_{2}+e_{2}\right)}} \pm \sqrt{-\frac{a_{2} \kappa^{2}+\omega}{c_{2}+d_{2}+e_{2}}} \operatorname{sech}\left[\sqrt{-\frac{a_{2} \kappa^{2}+\omega}{2 a_{2}}}\left(x+2 a_{2} \kappa t\right)\right]\right\} e^{i\left(-\kappa x+\omega t+\theta_{0}\right)} .
\end{aligned}
$$

The bright solitons are valid for the constraint (34) along with

$$
a_{l}\left(a_{l} \kappa^{2}+\omega\right)<0 .
$$

\section{Case 3:}

$$
\begin{aligned}
& \eta= \pm \sqrt{\frac{a_{l} \kappa^{2}+\omega}{a_{l}}}, \\
& A_{0}= \pm \sqrt{-\frac{a_{l} \kappa^{2}+\omega}{2\left(c_{l}+d_{l}+e_{l}\right)}}, \\
& A_{1}= \pm \sqrt{-\frac{a_{l} \kappa^{2}+\omega}{2\left(c_{l}+d_{l}+e_{l}\right)}}, \\
& B_{1}= \pm \sqrt{\frac{a_{l} \kappa^{2}+\omega}{2\left(c_{l}+d_{l}+e_{l}\right)},} \\
& b_{l}= \pm \sqrt{-\frac{9\left(a_{l} \kappa^{2}+\omega\right)\left(c_{l}+d_{l}+e_{l}\right)}{8}} .
\end{aligned}
$$

Substituting Eq. (40) with Eq. (10) into Eq. (28) yields the combo singular solitons

$$
\begin{aligned}
q(x, t) & =\left\{ \pm \sqrt{-\frac{a_{1} \kappa^{2}+\omega}{2\left(c_{1}+d_{1}+e_{1}\right)}} \pm \sqrt{-\frac{a_{1} \kappa^{2}+\omega}{2\left(c_{1}+d_{1}+e_{1}\right)}} \operatorname{coth}\left[\sqrt{\frac{a_{1} \kappa^{2}+\omega}{a_{1}}}\left(x+2 a_{1} \kappa t\right)\right]\right. \\
& \left. \pm \sqrt{-\frac{a_{1} \kappa^{2}+\omega}{2\left(c_{1}+d_{1}+e_{1}\right)}} \operatorname{csch}\left[\sqrt{\frac{a_{1} \kappa^{2}+\omega}{a_{1}}}\left(x+2 a_{1} \kappa t\right)\right]\right\} e^{i\left(-\kappa x+\omega t+\theta_{0}\right)},
\end{aligned}
$$




$$
\begin{aligned}
r(x, t) & =\left\{ \pm \sqrt{-\frac{a_{2} \kappa^{2}+\omega}{2\left(c_{2}+d_{2}+e_{2}\right)}} \pm \sqrt{-\frac{a_{2} \kappa^{2}+\omega}{2\left(c_{2}+d_{2}+e_{2}\right)}} \operatorname{coth}\left[\sqrt{\frac{a_{2} \kappa^{2}+\omega}{a_{2}}}\left(x+2 a_{2} \kappa t\right)\right]\right. \\
& \left. \pm \sqrt{-\frac{a_{2} \kappa^{2}+\omega}{2\left(c_{2}+d_{2}+e_{2}\right)}} \operatorname{csch}\left[\sqrt{\frac{a_{2} \kappa^{2}+\omega}{a_{2}}}\left(x+2 a_{2} \kappa t\right)\right]\right\} e^{i\left(-\kappa x+\omega t+\theta_{0}\right)} .
\end{aligned}
$$

The combo singular solitons are valid for the constraints (34) and (35).

\section{B. PERTURBED MODEL}

The coupled system derived from the equation (2) for birefringent fibers is:

$$
\begin{aligned}
& i q_{t}+a_{1} q_{x x}+b_{1} q \sqrt{|q|^{2}+|r|^{2}+q r^{*}+q^{*} r}+\left(c_{1}|q|^{2}+d_{1}|r|^{2}\right) q+e_{1} r^{2} q^{*} \\
& =i\left[\alpha_{1}\left(|q|^{2} q\right)_{x}+\beta_{1}\left(|r|^{2} r\right)_{x}+\left\{\lambda_{1}\left(|q|^{2}\right)_{x}+\gamma_{1}\left(|r|^{2}\right)_{x}\right\} q+\left(\mu_{1}|q|^{2}+\delta_{1}|r|^{2}\right) q_{x}\right], \\
& i r_{t}+a_{2} r_{x x}+b_{2} r \sqrt{|r|^{2}+|q|^{2}+r q^{*}+r^{*} q}+\left(c_{2}|r|^{2}+d_{2}|q|^{2}\right) r+e_{2} q^{2} r^{*} \\
& =i\left[\alpha_{2}\left(|r|^{2} r\right)_{x}+\beta_{2}\left(|q|^{2} q\right)_{x}+\left\{\lambda_{2}\left(|r|^{2}\right)_{x}+\gamma_{2}\left(|q|^{2}\right)_{x}\right\} r+\left(\mu_{2}|r|^{2}+\delta_{2}|q|^{2}\right) r_{x}\right],
\end{aligned}
$$

where the constants $\alpha_{l}, \beta_{l}, \lambda_{l}, \gamma_{l}, \mu_{l}$ and $\delta_{l}(l=1,2)$ are the coefficients of the nonlinear terms.

Inserting Eq. (24) into Eqs. (43) and (44) yields the real equation

$$
a_{l} \eta^{2} U_{l}^{\prime \prime}-\left(\omega+a_{l} \kappa^{2}\right) U_{l}+b_{l} U_{l}^{2}+b_{l} U_{l} U_{\tilde{l}}+\left(c_{l}-\kappa \alpha_{l}-\kappa \mu_{l}\right) U_{l}^{3}+\left(d_{l}+e_{l}-\kappa \delta_{l}\right) U_{l} U_{\tilde{l}}^{2}-\kappa \beta_{l} U_{\tilde{l}}^{3}=0
$$

and the imaginary equation

$$
\left(2 \lambda_{l}+3 \alpha_{l}+\mu_{l}\right) U_{l}^{2} U_{l}^{\prime}+2 \gamma_{l} U_{l} U_{\tilde{l}} U_{\tilde{l}}^{\prime}+\delta_{l} U_{\tilde{l}}^{2} U_{l}^{\prime}+3 \beta_{l} U_{\tilde{l}}^{2} U_{\tilde{l}}^{\prime}+\left(2 \kappa a_{l}+v\right) U_{l}^{\prime}=0,
$$

where $j=1,2$ and $\tilde{j}=3-j$. Equations (45) and (46) reduce to

$$
a_{l} \eta^{2} U_{l}^{\prime \prime}-\left(\omega+a_{l} \kappa^{2}\right) U_{l}+2 b_{l} U_{l}^{2}+\left(\begin{array}{c}
c_{l}+d_{l}+e_{l}-\kappa \alpha_{l} \\
-\kappa \beta_{l}-\kappa \mu_{l}-\kappa \delta_{l}
\end{array}\right) U_{l}^{3}=0
$$

and

$$
\left(2 \lambda_{l}+2 \gamma_{l}+3 \alpha_{l}+3 \beta_{l}+\mu_{l}+\delta_{l}\right) U_{l}^{2} U_{l}^{\prime}+\left(2 \kappa a_{l}+v\right) U_{l}^{\prime}=0
$$

by the constraint $U_{\tilde{l}}=U_{l}$. Equation (48) implies

$$
v=-2 a_{l} \kappa
$$

and

$$
2 \lambda_{l}+2 \gamma_{l}+3 \alpha_{l}+3 \beta_{l}+\mu_{l}+\delta_{l}=0 .
$$

By using the balancing principle in Eq. (47), Eq. (8) reduces to Eq. (28). Substituting Eq. (28) with Eq. (9) into Eq. (47) leads to 


\section{Case 1:}

$$
\begin{aligned}
& A_{1}= \pm \sqrt{\frac{\kappa^{2} a_{l}+\omega}{2\left(\begin{array}{c}
\kappa \alpha_{l}+\kappa \beta_{l}+\kappa \delta_{l} \\
+\kappa \mu_{l}-c_{l}-d_{l}-e_{l}
\end{array}\right)}}, \\
& A_{0}= \pm \sqrt{\frac{\kappa^{2} a_{l}+\omega}{2\left(\begin{array}{c}
\kappa \alpha_{l}+\kappa \beta_{l}+\kappa \delta_{l} \\
+\kappa \mu_{l}-c_{l}-d_{l}-e_{l}
\end{array}\right)}} \\
& \eta= \pm \sqrt{\frac{a_{l} \kappa^{2}+\omega}{4 a_{l}}}, \quad B_{1}=0 \\
& b_{l}= \pm \sqrt{\frac{9\left(\kappa^{2} a_{l}+\omega\right)\left(\begin{array}{c}
\kappa \alpha_{l}+\kappa \beta_{l}+\kappa \delta_{l} \\
+\kappa \mu_{l}-c_{l}-d_{l}-e_{l}
\end{array}\right)}{8}} .
\end{aligned}
$$

Plugging Eq. (51) with Eq. (10) into Eq. (28) leads to the dark solitons

$$
\begin{aligned}
& q(x, t)=\left\{ \pm \sqrt{\frac{\kappa^{2} a_{1}+\omega}{2\left(\begin{array}{c}
\kappa \alpha_{1}+\kappa \beta_{1}+\kappa \delta_{1} \\
+\kappa \mu_{1}-c_{1}-d_{1}-e_{1}
\end{array}\right)}} \pm \sqrt{\frac{\kappa^{2} a_{1}+\omega}{2\left(\begin{array}{c}
\kappa \alpha_{1}+\kappa \beta_{1}+\kappa \delta_{1} \\
+\kappa \mu_{1}-c_{1}-d_{1}-e_{1}
\end{array}\right)}}\right. \\
& \left.\times \tanh \left[\sqrt{\frac{a_{1} \kappa^{2}+\omega}{4 a_{1}}}\left(x+2 a_{1} \kappa t\right)\right]\right\} e^{i\left(-\kappa x+\omega t+\theta_{0}\right)}, \\
& r(x, t)=\left\{ \pm \sqrt{\frac{\kappa^{2} a_{2}+\omega}{2\left(\begin{array}{c}
\kappa \alpha_{2}+\kappa \beta_{2}+\kappa \delta_{2} \\
+\kappa \mu_{2}-c_{2}-d_{2}-e_{2}
\end{array}\right)}} \pm \sqrt{\frac{\kappa^{2} a_{2}+\omega}{2\left(\begin{array}{c}
\kappa \alpha_{2}+\kappa \beta_{2}+\kappa \delta_{2} \\
+\kappa \mu_{2}-c_{2}-d_{2}-e_{2}
\end{array}\right)}}\right. \\
& \left.\times \tanh \left[\sqrt{\frac{a_{2} \kappa^{2}+\omega}{4 a_{2}}}\left(x+2 a_{2} \kappa t\right)\right]\right\} e^{i\left(-\kappa x+\omega t+\theta_{0}\right)}
\end{aligned}
$$

and the singular solitons

$$
\begin{aligned}
& q(x, t)=\left\{ \pm \sqrt{\frac{\kappa^{2} a_{1}+\omega}{2\left(\begin{array}{c}
\kappa \alpha_{1}+\kappa \beta_{1}+\kappa \delta_{1} \\
+\kappa \mu_{1}-c_{1}-d_{1}-e_{1}
\end{array}\right)}} \pm \sqrt{\frac{\kappa^{2} a_{1}+\omega}{2\left(\begin{array}{c}
\kappa \alpha_{1}+\kappa \beta_{1}+\kappa \delta_{1} \\
+\kappa \mu_{1}-c_{1}-d_{1}-e_{1}
\end{array}\right)}}\right. \\
& \left.\times \operatorname{coth}\left[\sqrt{\frac{a_{1} \kappa^{2}+\omega}{4 a_{1}}}\left(x+2 a_{1} \kappa t\right)\right]\right\} e^{i\left(-\kappa x+\omega t+\theta_{0}\right)}
\end{aligned}
$$




$$
\begin{aligned}
& r(x, t)=\left\{ \pm \sqrt{\frac{\kappa^{2} a_{2}+\omega}{2\left(\begin{array}{c}
\kappa \alpha_{2}+\kappa \beta_{2}+\kappa \delta_{2} \\
+\kappa \mu_{2}-c_{2}-d_{2}-e_{2}
\end{array}\right)}} \pm \sqrt{\frac{\kappa^{2} a_{2}+\omega}{2\left(\begin{array}{c}
\kappa \alpha_{2}+\kappa \beta_{2}+\kappa \delta_{2} \\
+\kappa \mu_{2}-c_{2}-d_{2}-e_{2}
\end{array}\right)}}\right. \\
& \left.\times \operatorname{coth}\left[\sqrt{\frac{a_{2} \kappa^{2}+\omega}{4 a_{2}}}\left(x+2 a_{2} \kappa t\right)\right]\right\} e^{i\left(-\kappa x+\omega t+\theta_{0}\right)} .
\end{aligned}
$$

Once again, (52)-(53) give the perturbed dark-dark soliton pair in optics, while in fluids they are viewed as kink/antikink solitary waves or solitary waves. These solitons are valid for

$$
\begin{aligned}
\left(\begin{array}{c}
\kappa \alpha_{l}+\kappa \beta_{l}+\kappa \delta_{l} \\
+\kappa \mu_{l}-c_{l}-d_{l}-e_{l}
\end{array}\right)\left(\kappa^{2} a_{l}+\omega\right) & >0, \\
a_{l}\left(\kappa^{2} a_{l}+\omega\right) & >0 .
\end{aligned}
$$

Case 2:

$$
\begin{aligned}
& A_{0}= \pm \sqrt{\frac{\kappa^{2} a_{l}+\omega}{2\left(\begin{array}{c}
\kappa \alpha_{l}+\kappa \beta_{l}+\kappa \delta_{l} \\
+\kappa \mu_{l}-c_{l}-d_{l}-e_{l}
\end{array}\right)}}, \\
& B_{1}= \pm \sqrt{\frac{\kappa^{2} a_{l}+\omega}{\kappa \alpha_{l}+\kappa \beta_{l}+\kappa \delta_{l}}}+, \\
& \eta= \pm \sqrt{-\frac{a_{l} \kappa^{2}+\omega}{2 a_{l}}}, \quad A_{1}=0 \\
& b_{l}= \pm \sqrt{\frac{9\left(\kappa^{2} a_{l}+\omega\right)\left(\begin{array}{c}
\kappa \alpha_{l}+\kappa \beta_{l}+\kappa \delta_{l} \\
+\kappa \mu_{l}-c_{l}-d_{l}-e_{l}
\end{array}\right)}{8}} .
\end{aligned}
$$

Inserting Eq. (58) with Eq. (10) into Eq. (28) leads to the bright solitons

$$
\begin{aligned}
& q(x, t)=\left\{ \pm \sqrt{\frac{\kappa^{2} a_{1}+\omega}{2\left(\begin{array}{c}
\kappa \alpha_{1}+\kappa \beta_{1}+\kappa \delta_{1} \\
+\kappa \mu_{1}-c_{1}-d_{1}-e_{1}
\end{array}\right)}} \pm \sqrt{\frac{\kappa^{2} a_{1}+\omega}{\left(\begin{array}{c}
\kappa \alpha_{1}+\kappa \beta_{1}+\kappa \delta_{1} \\
+\kappa \mu_{1}-c_{1}-d_{1}-e_{1}
\end{array}\right)}}\right. \\
& \left.\times \operatorname{sech}\left[\sqrt{-\frac{a_{1} \kappa^{2}+\omega}{2 a_{1}}}\left(x+2 a_{1} \kappa t\right)\right]\right\} e^{i\left(-\kappa x+\omega t+\theta_{0}\right)}, \\
& r(x, t)=\left\{ \pm \sqrt{\frac{\kappa^{2} a_{2}+\omega}{2\left(\begin{array}{c}
\kappa \alpha_{2}+\kappa \beta_{2}+\kappa \delta_{2} \\
+\kappa \mu_{2}-c_{2}-d_{2}-e_{2}
\end{array}\right)}} \pm \sqrt{\frac{\kappa^{2} a_{2}+\omega}{\left(\begin{array}{c}
\kappa \alpha_{2}+\kappa \beta_{2}+\kappa \delta_{2} \\
+\kappa \mu_{2}-c_{2}-d_{2}-e_{2}
\end{array}\right)}}\right. \\
& \left.\times \operatorname{sech}\left[\sqrt{-\frac{a_{2} \kappa^{2}+\omega}{2 a_{2}}}\left(x+2 a_{2} \kappa t\right)\right]\right\} e^{i\left(-\kappa x+\omega t+\theta_{0}\right)} \text {. }
\end{aligned}
$$


The bright solitons are valid for the constraint (56) along with

$$
a_{l}\left(a_{l} \kappa^{2}+\omega\right)<0 .
$$

Case 3:

$$
\begin{aligned}
& A_{0}= \pm \sqrt{\frac{\kappa^{2} a_{l}+\omega}{2\left(\begin{array}{c}
\kappa \alpha_{l}+\kappa \beta_{l}+\kappa \delta_{l} \\
+\kappa \mu_{l}-c_{l}-d_{l}-e_{l}
\end{array}\right)}}, \\
& A_{1}= \pm \sqrt{\frac{\kappa^{2} a_{l}+\omega}{2\left(\begin{array}{c}
\kappa \alpha_{l}+\kappa \beta_{l}+\kappa \delta_{l} \\
+\kappa \mu_{l}-c_{l}-d_{l}-e_{l}
\end{array}\right)}} \\
& \eta= \pm \sqrt{\frac{a_{l} \kappa^{2}+\omega}{a_{l}}} \\
& B_{1}= \pm \sqrt{-\frac{\kappa^{2} a_{l}+\omega}{2\left(\begin{array}{c}
\kappa \alpha_{l}+\kappa \beta_{l}+\kappa \delta_{l} \\
+\kappa \mu_{l}-c_{l}-d_{l}-e_{l}
\end{array}\right)}} \\
& b_{l}= \pm \sqrt{\frac{9\left(\kappa^{2} a_{l}+\omega\right)\left(\begin{array}{c}
\kappa \alpha_{l}+\kappa \beta_{l}+\kappa \delta_{l} \\
+\kappa \mu_{l}-c_{l}-d_{l}-e_{l}
\end{array}\right)}{8}}
\end{aligned}
$$

Substituting Eq. (62) with Eq. (10) into Eq. (28) yields the combo singular solitons

$$
\begin{aligned}
& q(x, t)=\left\{ \pm \sqrt{\frac{\kappa^{2} a_{1}+\omega}{2\left(\begin{array}{c}
\kappa \alpha_{1}+\kappa \beta_{1}+\kappa \delta_{1} \\
+\kappa \mu_{1}-c_{1}-d_{1}-e_{1}
\end{array}\right)}} \pm \sqrt{\frac{\kappa^{2} a_{1}+\omega}{2\left(\begin{array}{c}
\kappa \alpha_{1}+\kappa \beta_{1}+\kappa \delta_{1} \\
+\kappa \mu_{1}-c_{1}-d_{1}-e_{1}
\end{array}\right)}} \operatorname{coth}\left[\sqrt{\frac{a_{1} \kappa^{2}+\omega}{a_{1}}\left(x+2 a_{1} \kappa t\right)}\right]\right. \\
& \left. \pm \sqrt{\frac{\kappa^{2} a_{1}+\omega}{2\left(\begin{array}{c}
\kappa \alpha_{1}+\kappa \beta_{1}+\kappa \delta_{1} \\
+\kappa \mu_{1}-c_{1}-d_{1}-e_{1}
\end{array}\right)}} \times \operatorname{csch}\left[\sqrt{\frac{a_{1} \kappa^{2}+\omega}{a_{1}}}\left(x+2 a_{1} \kappa t\right)\right]\right\} e^{i\left(-\kappa x+\omega t+\theta_{0}\right)} \\
& r(x, t)=\left\{ \pm \sqrt{\frac{\kappa^{2} a_{2}+\omega}{2\left(\begin{array}{c}
\kappa \alpha_{2}+\kappa \beta_{2}+\kappa \delta_{2} \\
+\kappa \mu_{2}-c_{2}-d_{2}-e_{2}
\end{array}\right)}} \pm \sqrt{\frac{\kappa^{2} a_{2}+\omega}{2\left(\begin{array}{c}
\kappa \alpha_{2}+\kappa \beta_{2}+\kappa \delta_{2} \\
+\kappa \mu_{2}-c_{2}-d_{2}-e_{2}
\end{array}\right)}} \operatorname{coth}\left[\sqrt{\frac{a_{2} \kappa^{2}+\omega}{a_{2}}\left(x+2 a_{2} \kappa t\right)}\right]\right. \\
& \left. \pm \sqrt{\frac{\kappa^{2} a_{2}+\omega}{2\left(\begin{array}{c}
\kappa \alpha_{2}+\kappa \beta_{2}+\kappa \delta_{2} \\
+\kappa \mu_{2}-c_{2}-d_{2}-e_{2}
\end{array}\right)}} \times \operatorname{csch}\left[\sqrt{\frac{a_{2} \kappa^{2}+\omega}{a_{2}}}\left(x+2 a_{2} \kappa t\right)\right]\right\} e^{i\left(-\kappa x+\omega t+\theta_{0}\right)} .
\end{aligned}
$$

The combo singular soliton are valid for the constraints (56) and (57).

\section{CONCLUSIONS}

This paper accrues soliton solutions to fibers with QC nonlinearity in the presence of CD. A full spectrum of solitons, both single- and dual-form are exhi- bited. The Hamiltonian type perturbation terms that are incorporated are of maximum allowable intensity. The absolute maximum or critical value of the intensity is yet to be discovered. The Benjamin-Fier instability analysis is not yet studied for the model. Moreover, 
the study needs to be extended later with quadraticcubic nonlinear structures of the refractive index. In that case, the integrability issues will be addressed for the perturbed equation, and the corresponding soliton solutions along with the conservation laws for the model will be displayed. Additionally, the model has to be extended to address DWDM topologyand the formulated model will also be studied. Several additional integration schemes will also be implemented [11-16]. The readers are suggested to stay tuned!
[1] A. R. Adem et al., Phys. Lett. A 384, 126606 (2020); https://doi.org/10.1016/j.physleta.2020.126606.

[2] M. Asma, W. A. M. Othman, B. R. Wong, A. Biswas, Proc. Roman. Acad. A 18, 331 (2017).

[3] A. Biswas, M. Ekici, A. Sonmezoglu, M. Belic, Optik 178, 59 (2019); https://doi.org/10.1016/j.ijleo.20 18.09.159.

[4] A. Biswas, M. Ekici, A. Sonmezoglu, M. Belic, Optik 178, 117 (2019); https://doi.org/10.1016/j.ijleo. 2018.09.154.

[5] A. Biswas, M. Ekici, A. Sonmezoglu, M. Alfiras, Optik 178, 178 (2019); https://doi.org/10.1016/j.ijleo. 2018.09.180.

[6] A. Biswas et al., Chin. J. Phys. 56, 1990 (2018); https: //doi.org/10.1016/j.cjph.2018.09.009.

[7] J. Fujioka et al., Chaos 21, 033120 (2011); https://do i.org/10.1063/1.3629985.

[8] K. Hayata, M. Koshiba, J. Opt. Soc. Am. B 11, 2581 (1994); https://doi.org/10.1364/JOSAB.11.002581.

[9] S. Khan, Optik 212, 164706 (2020); https://doi.org/ 10.1016/j.ijleo.2020.164706.

[10] E. V. Krishnan et al., Chin. J. Phys. 60, 632 (2019); https://doi.org/10.1016/j.cjph.2019.06.002.
[11] N. A. Kudryashov, Optik 189, 42 (2019); https://doi. org/10.1016/j.ijleo.2019.05.069.

[12] N. A. Kudryashov, E. V. Antonova, Optik 217, 164881 (2020); https://doi.org/10.1016/j.ijleo.20 20.164881.

[13] N. A. Kudryashov, Optik 206, 163550 (2020); https: //doi.org/10.1016/j.ijleo.2019.163550.

[14] N. A. Kudryashov, Chaos Solitons Fractals 141, 110325 (2020); https://doi.org/10.1016/j.chaos. 20 20.110325.

[15] N. A. Kudryashov, Chaos Solitons Fractals 140, 110202 (2020); https://doi.org/10.1016/j.chaos.20 20.110202 .

[16] N. A. Kudryashov, Chin. J. Phys. 66, 401 (2020); https: //doi.org/10.1016/j.cjph.2020.06.006.

[17] H. Triki et al., Opt. Commun. 437, 392 (2019); https: //doi.org/10.1016/j.optcom. 2018.12.074.

[18] E. M. E. Zayed et al., Optik 202, 163620 (2020); https: //doi.org/10.1016/j.ijleo.2019.163620.

[19] E. M. E. Zayed et al., Optik 203, 163993 (2020); https: //doi.org/10.1016/j.ijleo.2019.163993.

[20] E. M. E. Zayed et al., Phys. Lett. A. 384, 126456 (2020); https://doi.org/10.1016/j.physleta.2020.126456.

\section{ОПТИЧНЕ СОЛІТОННЕ ЗБУРЕННЯ ТА ПОЛЯРИЗАЦІЯ 3 КВАДРАТИЧНО-КУБІЧНОЮ НЕЛІНІЙНІСТЮ В ПІДХОДІ РІВНЯННЯ СИНУС-ГОРДОНА}

Я. Йилдирим ${ }^{1}$, Е. Топкара ${ }^{2}$, А. Бісвас ${ }^{3,4,5,6}$, Г. Трикі ${ }^{7}$, М. Екіджі ${ }^{8}$, П. Гугтілла ${ }^{3}$, С. Хан $^{3}$, М. Р. Беліч ${ }^{9}$

${ }^{1}$ Кафедра математики факулътету гуманітарних і природничих наук, Університет Близъкого Сходу, 99138, Нікосія, Kinp,

${ }^{2}$ Кафедра математики, Університет Г'юстона-Тіллотсона, Остін, Техас-78702, США,

${ }^{3}$ Кафедра фізики, хімій та математики, Університет Алабами Аєм, Нормаль, Алабама, 35762-4900, США,

${ }_{4}^{4}$ Дослідницька група з математичного моделювання та прикладних обчислень (ММАС),

Департамент математики, Університет короля Абдулазіза, Джидда, 21589, Саудівсвка Аравія,

${ }^{5}$ Кафедра прикладной математики, Націоналъний дослідницъкий ядерний університет, Каширсъке шосе, Москва, 115409, Російсъка Федерачія,

${ }^{6}$ Кафедра математики та прикладной математики, Університет наук про здоров'я Сефако Макљато, Медунса, 0204, Преторія, ПАР,

${ }^{7}$ Лабораторія радіаційної фізики, Фізичне відділення, Факультет природничих наук, Університет Баджі Мохтара, 23000, Аннаба, Алжир,

${ }^{8}$ Кафедра математики факультету наук і мистецтв, Університет Йозґат Бозок, 66100, Йозґат, Туреччина,

${ }^{9}$ Інститут фізики в Белграді, Прељ'ревіча, 118, 11080 Земун, Сербіл

У статті відтворено повний спектр оптичних солітонів із квадратично-кубічною нелінійністю у волокнах, що зберігають поляризацію, а також у волокнах із двозаломленням. Доданки збурення мають повну інтенсивність. Таку задачу можна розв'язати в підході рівняння синус-Гордона. Також є відповідні обмежувальні умови, які гарантують наявність таких солітонів.

Ключові слова: солітони, поляризація, збурення, квадратично-кубічна нелінійність. 